\title{
Localised retinal nerve fibre layer defects in chronic experimental high pressure glaucoma in rhesus monkeys
}

\author{
Jost B Jonas, Sohan Singh Hayreh
}

\begin{abstract}
Aim-To evaluate prospectively in an experimental model of chronic high pressure glaucoma whether the concept of a mainly diffuse pattern of optic nerve damage holds true for high pressure glaucoma.

Methods-The study comprised nine eyes of nine rhesus monkeys (Macaca mulatta) with a mean age of 17.7 (SD 3.1) years (range 13-23 years). Experimental glaucoma was produced by multiple applications of argon laser to the trabecular meshwork. Applanation tonometry was regularly performed and fundus photographs, which were taken serially, were used for retinal nerve fibre layer (RNFL) assessment and morphometric optic disc analysis. Six monkeys, in which arterial hypertension and atherosclerosis had additionally been induced several years before elevation of intraocular pressure, did not show any sign of diffuse loss or localised defects of the RNFL before initiation of glaucoma.
\end{abstract}

Results-Compared with the same eyes at baseline, localised RNFL defects had developed in eight $(89 \%)$ eyes. It included all three eyes $(100 \%)$ of the monkeys without arterial hypertension/arteriosclerosis, and five of the six monkeys (83\%) with arterial hypertension/arteriosclerosis. Four eyes had multiple localised RNFL defects. In all eyes, diffuse RNFL loss was additionally present.

Conclusions-Besides diffuse loss of RNFL, localised RNFL defects were present in almost all eyes of monkeys with chronic experimental high pressure glaucoma. Challenging the concept that a mostly diffuse type of optic neuropathy occurs in high pressure glaucoma, the results suggest that, in high pressure glaucoma, at least a mixture of localised and diffuse pattern of optic nerve damage prevails.

(Br F Ophthalmol 1999;83:1291-1295)

Ophthalmology and

Visual Sciences,

College of Medicine,

University of Iowa,

Iowa City, IA 52242,

USA

S S Hayreh

Correspondence to:

Dr Jost Jonas, University Eye

Hospital, Schwabachanlage

6, 91054 Erlangen, Germany

Accepted for publication 2 July 1999

The glaucomas are a heterogeneous group of diseases which have in common the glaucomatous loss of optic nerve fibres. They show a multitude of morphological and psychophysical changes, such as a decrease in neuroretinal rim and secondary enlargement of the optic cup, deepening of the optic cup, enlargement of parapapillary atrophy, diffuse and focally accentuated reduction in the calibre of the reti- nal arterioles, loss of retinal nerve fibre layer, reduced differential light sensitivity, and decreased flicker fusion frequency, to mention only some of the changes. ${ }^{12}$ In all these alterations, mostly localised changes can be differentiated from mainly diffuse changes, such as neuroretinal rim notching versus diffuse rim loss, ${ }^{3}$ presence or absence of splinter-shaped optic disc haemorrhages, ${ }^{4}$ sharply demarcated localised defects in the retinal nerve fibre layer in contrast with diffuse loss of retinal nerve fibre layer, ${ }^{5}$ and circumscribed deep scotomata in the visual field versus diffuse perimetric loss. ${ }^{6}$ Previous studies have suggested that these localised alterations and diffuse changes are unevenly distributed between the various types of glaucomas, with localised changes dominating in patients with the focal type of normal pressure glaucoma, and diffuse alterations prevailing in eyes with high pressure glaucoma. ${ }^{7-16}$ This had led to the hypothesis that two pathomechanisms, a localised one and a diffuse one, may have different roles in the various types of glaucoma ${ }^{17}$; that in the focal type of normal pressure glaucoma, mainly a localised type of optic nerve atrophy dominates, while in high pressure glaucoma, the optic nerve is mostly diffusely damaged.

The purpose of the present longitudinal study was to evaluate whether, indeed, high pressure glaucoma is associated with a mainly diffuse type of optic nerve damage. We prospectively examined rhesus monkeys in which a secondary high pressure glaucoma had been produced experimentally.

\section{Material and methods}

The study was conducted in nine eyes of nine rhesus monkeys (Macaca mulatta) with a mean age of 17.7 (SD 3.1) years (range 13-23 years). At Iowa City, experimental glaucoma was produced by multiple applications of argon laser to the trabecular meshwork. The study design complied with the National Institute of Health as well as University of Iowa's institutional guidelines for the care and use of laboratory animals. In six of the nine monkeys, atherosclerosis was produced by feeding the animals a special atherogenic diet (consisting of $1 \mathrm{mg}$ cholesterol per calorie ( $0.8 \%$ by weight) and $43 \%$ of total calories as fat) continuously for many years, and all of them showed marked elevation of the serum lipids. ${ }^{18}$ Chronic arterial hypertension was produced by modified Goldblatt's procedure ${ }^{19}$ and the hypertension persisted for several years. Then experimental glaucoma was produced. 
All eyes showed no abnormality on a detailed ophthalmic evaluation before the production of elevated intraocular pressure. This holds true especially for the six monkeys in which arterial hypertension and atherosclerosis had additionally been induced several years before elevation of the intraocular pressure. They did not present any sign of diffuse loss or localised defects of the retinal nerve fibre layer before initiation of glaucoma. All animals were followed by intraocular pressure measurement with Goldmann applanation tonometry, ophthalmoscopic examinations, fluorescein fundus angiography, and stereoscopic colour fundus photography. These clinical examinations, performed under ketamine anaesthesia (8-10 $\mathrm{mg} / \mathrm{kg}$ body weight) on both the glaucomatous and the fellow normal eyes, were carried out before the application of laser photocoagulation and serially thereafter during the follow up period. Mean length of the follow up period was 25.7 (SD 13.4) months (median 21 months; range 9-46 months).

Before the application of laser photocoagulation, intraocular pressure was measured three times on three different days to establish a baseline for each eye. During the follow up period, after the eyes had developed elevated intraocular pressure (sustained intraocular pressure of $>21 \mathrm{~mm} \mathrm{Hg}$ ), the frequency of intraocular pressure measurements depended upon the level of intraocular pressure in each eye; the higher the intraocular pressure, the more frequently it was measured-that is, two to three times a week when it was $>60 \mathrm{~mm} \mathrm{Hg}$, weekly when in the 40 s or 50 s, and monthly when $<40 \mathrm{~mm} \mathrm{Hg}$. The objective was to maintain an intraocular pressure between 30 and 40 $\mathrm{mm} \mathrm{Hg}$, to mimic the clinical situation of moderate ocular hypertension. Since, with the argon laser trabecular application, it is impossible to achieve a desired level of intraocular pressure on a long term basis, the intraocular pressure often went higher than the desired levels. We had to use ocular hypotensive drops in five eyes to maintain the desired level of intraocular pressure. Three eyes received timolol drops, one eye timolol and acepromazine drops, and one eye received timolol, acepromazine, and phospholine iodide drops.

The morphological evaluation of the colour fundus photographs was performed in Erlangen (Germany). Mixed together with the fundus diapositives of other monkeys, the photographs taken at baseline of the present study together with the photographs taken at the end of the study were evaluated in a masked fashion. The slides were projected with a magnification of 15 times after maximal defocusing of the projector. The area of the blurred image of the optic disc was covered, then the projector refocused and the retinal nerve fibre layer evaluated. A localised defect was defined as a wedge-shaped and not spindle-like defect, running towards or touching the optic disc border for not more than $60^{\circ}$ of the optic disc circumference. A pseudodefect was defined as a spindle-like but not wedge-shaped narrow dark area in the retinal nerve fibre layer. The retinal nerve fibre layer was assessed independently of the optic disc examination, without knowledge of the history and the morphometric optic disc data. In all eyes, visibility of the retinal nerve fibre layer was not totally reduced so that localised retinal nerve fibre layer defects could, at least theoretically, be detected.

In an independent second step, the same transparencies were again projected, and the outlines of the optic cup, optic disc, peripapillary scleral ring, and alpha and beta zone of the parapapillary atrophy were plotted on paper and morphometrically analysed. The optic cup was defined on the basis of contour and not of pallor. The border of the optic disc was identical with the inner side of the peripapillary scleral ring. The parapapillary atrophy was differentiated into a peripheral alpha zone with irregular pigmentation, and a central beta zone with visible Bruch's membrane and visible large choroidal vessels. ${ }^{20}$ The method has already been described in detail. Since the magnification of the optic disc photographs varied according to the period of the study and the fundus camera used, all disc measurements of the same monkey were adapted to the photographic magnification of the first photograph. Because keratometric readings and refractometry had not been performed for all monkeys included in the study, the optic disc measurements were expressed in relative size units.

\section{Results}

Compared with the same eyes at baseline, localised defects of the retinal nerve fibre layer had developed in eight eyes (89\%) (Figs 1, 2). It included all three eyes $(100 \%)$ of the monkeys without arterial hypertension/ atherosclerosis, and five of the six monkeys
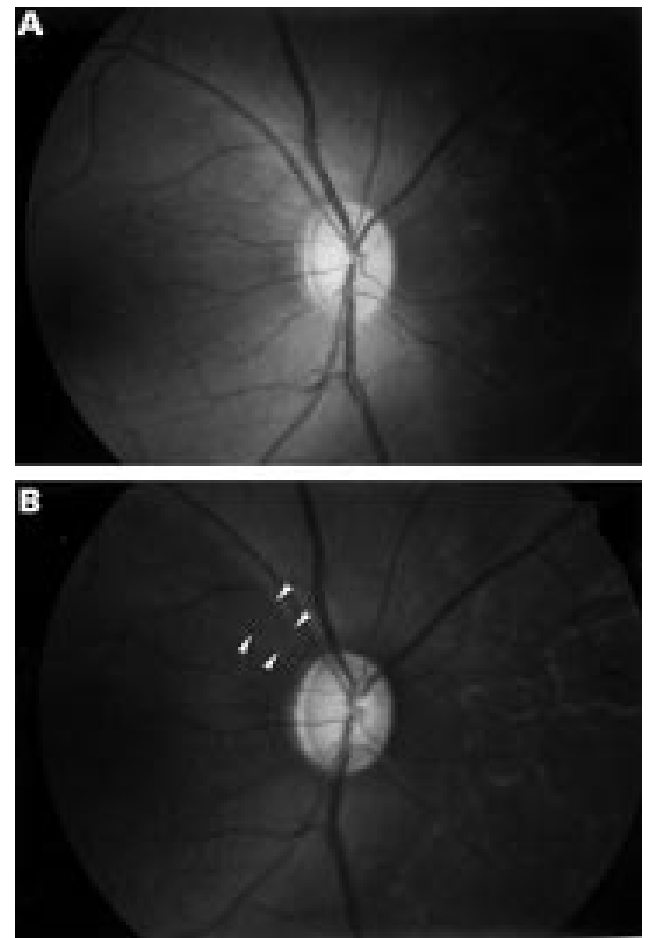

Figure 1 Fundus photographs of monkey before $(A)$ and after (B) experimental elevation of intraocular pressure. Arrows: localised retinal nerve fibre layer defects. 

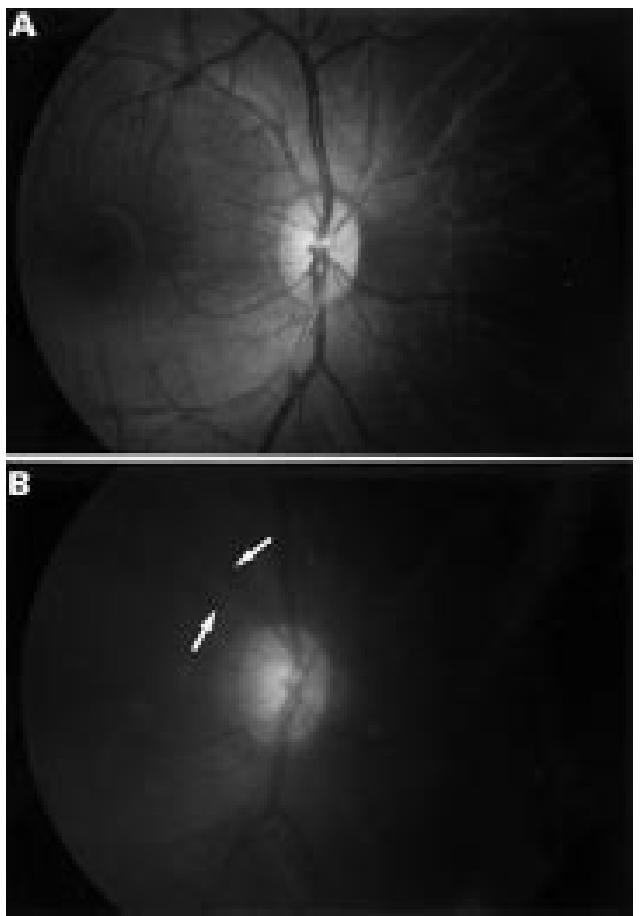

Figure 2 Fundus photographs of monkey before $(A)$ and after (B) experimental elevation of intraocular pressure. Arrows: localised retinal nerve fibre layer defects.

(83\%) with arterial hypertension/ atherosclerosis. Four eyes had multiple localised retinal nerve fibre layer defects. Compared with the photographs taken at baseline, diffuse loss of the retinal nerve fibre layer was also present in all eyes. At baseline before induction of elevated intraocular pressure, all eyes had been free of localised retinal nerve fibre layer defects or a diffuse loss of the retinal nerve fibre layer.

All localised retinal nerve fibre layer defects were detected in the superior temporal sector and/or the inferior temporal sector of the fundus. No defects were seen in the temporal horizontal fundus sector or in the nasal fundus region.

In all of the eyes with localised defects of the retinal nerve fibre layer, the neuroretinal rim was significantly smaller $(0.24(0.19)$ relative $\mathrm{mm}^{2}$ versus $0.68(0.08)$ relative $\mathrm{mm}^{2} ; \mathrm{p}=0.012$; Wilcoxon sign rank test) and parapapillary atrophy as sum of alpha zone and beta zone was significantly larger $(0.49(0.14)$ relative $\mathrm{mm}^{2}$ versus $0.40(0.11)$ relative $\mathrm{mm}^{2} ; \mathrm{p}=0.012$; Wilcoxon sign rank test) than at baseline.

The number of eyes without detectable localised retinal nerve fibre layer defects (one eye) was statistically too small to address the question whether the beta zone of parapapillary atrophy influenced the development of localised retinal nerve fibre layer defects.

\section{Discussion}

After the first description of the ophthalmoscopic appearance of the retinal nerve fibre layer by Vogt in $1913^{21}$ and its "rediscovery" by Hoyt and coworkers in 1972 and 1973, ${ }^{22}{ }^{23}$ alterations of the retinal nerve fibre layer were differentiated into a diffuse loss and a localised dropout of retinal nerve fibre bundles. ${ }^{5}$ Localised defects of the retinal nerve fibre layer are defined as wedge-shaped and not spindle-like defects, running towards or touching the optic disc border. If they are very deep, they can have a broad basis at the temporal raphe of the fundus. Typically occurring in eyes with glaucoma, ${ }^{24}$ they can also be found in eyes with an atrophy of the optic nerve for reasons other than glaucoma, such as optic disc drusen, toxoplasmic chorioretinal scars, ischaemic retinopathies with cotton wool spots of the retina, after long standing papilloedema or optic neuritis due to multiple sclerosis, to mention some examples. ${ }^{25}$ Since the localised retinal nerve fibre layer defects are not present in normal eyes, they almost always signify a pathological abnormality leading to a high specificity as diagnostic marker. ${ }^{24}$ In glaucomatous eyes, localised retinal nerve fibre layer defects increase significantly in frequency from an "early" glaucoma stage to a stage with medium advanced glaucomatous damage, and then decrease again to a stage with very marked glaucomatous changes. ${ }^{24}$ In eyes with very advanced optic nerve damage, they are usually no longer detectable owing to the pronounced loss of nerve fibres in all fundus sectors. In the vicinity of localised retinal nerve fibre layer defects at the optic disc border, one often finds notches of the neuroretinal rim, and sometimes an optic disc haemorrhage. ${ }^{2326}$ Localised retinal nerve fibre layer defects are often detected 6-8 weeks after an optic disc bleeding. ${ }^{26}$ With respect to different sectors of the fundus, localised retinal nerve fibre layer defects are most often found in the inferior temporal sector followed by the superior temporal sector. In the nasal fundus region, localised retinal nerve fibre layer defects are only rarely seen. ${ }^{24}$ This may be due to the fact that there is regional variation in the thickness of the retinal nerve fibre layer; it is thicker in the superior temporal and inferior temporal regions than in the nasal region. Ophthalmoscopically, therefore, the normal retinal nerve fibre layer is less detectable in the nasal fundus than in the inferior temporal and superior temporal regions. ${ }^{27}$ In areas in which the retinal nerve fibre layer physiologically is thin, localised defects are harder to find than in areas with a thick retinal nerve fibre layer. It is unclear whether the morphology of the lamina cribrosa (larger pores in the inferior and superior sectors and smaller pores in the temporal and nasal regions) have a role in the development of localised retinal nerve fibre layer defects. ${ }^{28}$

Localised retinal neve fibre layer defects have been considered as features of a localised pattern of glaucomatous optic nerve damage. Previous clinical studies have suggested that, owing to the relatively low frequency of localised retinal nerve fibre layer defects in combination with a low prevalence of detectable disc haemorrhages and concentric loss of neuroretinal rim, patients with high pressure glaucoma, in contrast with patients with the focal type of normal pressure glaucoma, exhibit a mostly diffuse type of glaucomatous optic nerve damage. ${ }^{7-16}$ The results of the 
present experimental study, however, contradict this finding. The monkeys with purely high pressure glaucoma in our prospective study showed, besides a diffuse loss of retinal nerve fibre layer, typical localised retinal nerve fibre layer defects. This suggests that, in the high pressure type of glaucoma, at least a mixture of localised and diffuse pattern of optic nerve damage occurs.

The question then arises whether a purely diffuse pattern or purely localised pattern or a mixture of localised and diffuse optic nerve damage prevails in the various glaucomas. Several aspects have to be taken into account. The diameter of a single retinal nerve fibre of about $1 \mu \mathrm{m}$ is too small to be ophthalmoscopically detectable. Upon ophthalmoscopy, only bundles of retinal nerve fibres can be seen. ${ }^{29}$ This explains why, in eyes with advanced optic nerve damage, no retinal nerve fibre layer is ophthalmoscopically visible although visual function is still partially preserved. Correspondingly, there is the possibility that all glaucomatous optic nerve fibre loss occurs in a localised pattern; however, many localised nerve fibre layer defects may be too small to be detected ophthalmoscopically individually (localised "minidefects"). Because the individual losses are so small, the loss may become visible only when many of them combine. This loss may appear to be diffuse when in fact it is localised ("pseudo diffuse loss"). This is supported by experimental studies which have shown that localised retinal nerve fibre layer defects can ophthalmoscopically be detected only if more than $50 \%$ of the thickness of the retinal nerve fibre layer are lost. ${ }^{30}$ It may suggest that the ophthalmoscopically diffuse type and the ophthalmoscopically localised type of retinal nerve fibre layer loss are the ophthalmoscopic manifestations of the same mechanism. Correspondingly, the diffuse retinal nerve fibre layer loss in eyes with very advanced glaucomatous optic nerve damage may be the result of broadening localised retinal nerve fibre layer defects. That explains the decreased frequency of localised retinal nerve fibre layer defects in eyes with far advanced or absolute glaucoma compared with eyes with medium advanced optic nerve atrophy. ${ }^{24}$

In several aspects, the present monkey study confirms previous studies on patients with glaucoma. As in preceding clinical studies ${ }^{24}$ no localised retinal nerve fibre layer defects were observed in the normal eyes of the present investigation, explaining the high specificity of localised retinal nerve fibre layer defects in indicating optic nerve damage. In the present monkey study, the localised retinal nerve fibre layer defects were found in the superior temporal and inferior temporal fundus regions. None was detected in the temporal horizontal fundus area nor in the nasal part of the fundus. A similar finding has been described for the location of localised retinal nerve fibre layer defects in patients with glaucoma. ${ }^{24}$ The question arises whether the localised retinal nerve fibre layer defects were more often observed in the inferior temporal and superior temporal sectors because these regions physiologically have a greater thickness ${ }^{31} 32$ and better visibility of the retinal nerve fibre layer ${ }^{27}$ than other fundus areas, or because the nerve fibres in the inferior temporal and superior temporal fundus regions are preferentially damaged by circumscribed mechanisms.

There are some factors possibly limiting the present study. Five of the nine monkey eyes received antiglaucomatous drops to reduce the intraocular pressure. Although one cannot completely exclude the possibility that these drops might have influenced the results of the study, it is rather unlikely. Furthermore, localised retinal nerve fibre layer defects were detected in eyes which received antiglaucomatous treatment, as well as in eyes which were not under treatment. The sample size of nine eyes included in the study is relatively small. The purpose of the study, however, was to evaluate whether eyes with experimental high pressure glaucoma can develop localised retinal nerve fibre layer defects. It was not the goal of the investigation to determine the percentage of eyes which developed or which did not develop localised retinal nerve fibre layer defects. For the qualitative statement that eyes with experimental high pressure glaucoma can show localised retinal nerve fibre layer defects, the sample size of nine eyes may be sufficient. Colour photographs instead of red-free photographs were taken. It might have prevented the examiner from detecting localised retinal nerve fibre layer defects at the baseline of the study. Considering the increased age of the animals at the end of the study, however, the chance of missing a localised retinal nerve fibre layer defect is higher for the photographs taken at the end of the study than for the photographs taken at baseline. One may postulate that both effects might have compensated each other. Since fundus photography with monkeys is more difficult than with patients, some of the fundus photographs were slightly out of focus and showed another colour. If these technical difficulties might have prevented the discovery of some localised retinal nerve fibre layer defects, it may only serve to underline the result of the study that many eyes with high pressure glaucoma can develop localised retinal nerve fibre layer defects. Instead of sophisticated imaging techniques such as confocal laser scanning laser tomography, laser polarimetry of the retinal nerve fibre layer, or optical coherence tomography we used conventional fundus photography. This lack of new technology may again only serve to underline the result of the study that eyes with experimental high pressure glaucoma can develop localised retinal nerve fibre layer defects. With the newer techniques one might have detected a localised defect even in the ninth eye in which no defect could be seen on conventional fundus photographs.

In conclusion, the present prospective longitudinal study on monkeys with high pressure glaucoma suggests that, in contrast with previous findings, optic nerve damage in high pressure glaucoma consists of at least a mixture of an ophthalmoscopically localised pattern and an ophthalmoscopically diffuse pattern which 
both may be due to a similar pathomechanism. Future longitudinal studies on patients with high pressure glaucoma may reveal if this finding holds true also for humans, and if the ophthalmoscopically diffuse pattern of optic nerve damage found in some eyes with glaucoma is only the result of merging of localised "minidefects" of the retinal nerve fibre layer.

Dr S S Hayreh is a Research to Prevent Blindness senior scientific investigator.

Supported by grant EY-1576 from the US National Institutes of Health, in part by unrestricted grants from Research to Prevent Blindness, Inc, New York, USA, and by Deutsche Forschungsgemeinschaft (SFB 539).

1 Airaksinen PJ, Tuulonen A, Werner EB. Clinical evaluation of the optic disc and retinal nerve fiber layer. In: Ritch R, Shields MB, Krupin T, eds. The glaucomas. St Louis: Mosby, 1989:467-94.

2 Horn F, Jonas JB, Jünemann A, et al. The full-field flicker test in early diagnosis of chronic open-angle glaucoma. $\mathrm{Am}$ test in early diagnosis of chronic

3 Javitt JC, Spaeth GL, Katz LJ, et al. Acquired pits of the optic nerve. Ophthalmology 1990;97:1038-44

4 Drance SM, Fairclough M, Butler DM, et al. The importance of disc hemorrhage in the prognosis of chronic importance of disc hemorrhage in the prognosis of chron
open-angle glaucoma. Arch Ophthalmol 1977;95:226-8.

5 Airaksinen PJ, Drance SM, Douglas GR, et al. Diffuse and localised nerve fiber loss in glaucoma. Am $\mathcal{F}$ Ophthalmo 1984;98:566-71.

6 Drance SM, Douglas GR, Airaksinen PJ, et al. Diffuse visual field loss in chronic open-angle and low-tension glaucoma Am $\mathcal{F}$ Ophthalmol 1987;104:577-80.

7 Spaeth GL, Hitchings RA, Sivalingam E. The optic disc in glaucoma: pathogenetic correlation of five patterns of cupping in chronic open-angle glaucoma. Trans Am Acad Ophthalmol Otolaryngol 1976;81:217-23.

8 Drance SM, Airaksinen PJ, Price M, et al. The correlation of functional and structural measurements in glaucoma patients and normal subjects. Am f Ophthalmol 1986;102: 612-6.

9 Geijssen HC, Greve EL. Focal ischaemic normal pressure glaucoma versus high pressure glaucoma. Doc Ophthalmol glaucoma versus high

10 Caprioli J. Correlation between disc appearance and type of glaucoma. In: Varma R, Spaeth GL, eds. The optic nerve in glaucoma. Philadelphia: Lippincott, 1993:91-8.

11 Spaeth GL. A new classification of glaucoma including focal glaucoma. Surv Ophthalmol 1994;38:S9-17.

12 Spaeth GL, Katz LJ, Terebuh AK. Managing glaucoma on the basis of tissue damage: a therapeutic approach based largely on the appearance of the optic disc. In: Krieglstein GK, ed. Glaucoma update V. Heidelberg: Kaden-Verlag 1995:118-23.

13 Nicolela MT, Drance SM. Various glaucomatous optic nerve appearances: clinical correlations. Ophthalmology 1996;103:640-9.
14 Jonas JB, Gründler AE. Optic disc morphology in "age-related sclerotic glaucoma". Graefes Arch Clin Exp "age-related sclerotic glauco

15 Jonas JB, Gründler AE. Optic disc morphology in juvenile primary open-angle glaucoma. Graefes Arch Clin Exp Ophthalmol 1996;234:750-4.

16 Jonas JB, Dichtl A. Optic disc morphology in myopic primary open-angle glaucoma. Graefes Arch Clin Exp Ophthalmol 1997;235:627-33.

17 Drance SM. Mechanisms of optic nerve damage in glaucoma. Fortschr Ophthalmol 1988;85:611-3.

18 Hayreh SS, Piegors DJ, Heistad DD. Serotonin induced constriction of ocular arteries in atherosclerotic monkeys: mplications for ischemic disorders of retina and optic nerve head. Arch Ophthalmol 1997;115:220-8.

19 Hayreh SS, Servais GE, Virdi PS, et al. Fundus lesions in malignant hypertension III. Arterial blood pressure, biochemical, and fundus changes. Ophthalmology 1986;93: $45-59$.

20 Hayreh SS, Jonas JB, Zimmerman MB. Parapapillary chorioretinal atrophy in chronic high-pressure experimental glaucoma in rhesus monkeys. Invest Ophthalmol Vis Sci glaucoma in rhesus

21 Vogt A. Herstellung eines gelbblauen Lichtfiltrates, in welchem die Makula centralis in vivo in gelber Farbe erscheint, die Nervenfasern der Netzhaut und andere feine Einzelheiten derselben sichtbar werden und der Grad der Gelbfärbung der Linse ophthalmoskopisch nachweisbar ist. Graefes Arch Clin Exp Ophthalmol 1913;84:293-311.

22 Hoyt WF, Frisén LL, Newman NM. Funduscopy of nerve fiber layer defects in glaucoma. Invest Ophthalmol 1973;12: $814-29$

23 Hoyt WF, Schlicke B, Eckelhoff RJ. Funduscopic appearance of a nerve fiber bundle defect. Br F Ophthalmol 1972; 56:577-83.

24 Jonas JB, Schiro D. Localized wedge shaped defects of the retinal nerve fiber layer in glaucoma. Br f Ophthalmol 1994; 78:285-90.

25 Jonas JB, Schiro D. Localized retinal nerve fiber layer defects in nonglaucomatous optic nerve atrophy. Graefes Arch Clin Exp Ophthalmol 1994;232:759.

26 Airaksinen PJ, Mustonen E, Alanku HI. Optic disc haemorrhages precede retinal nerve fibre laver defects in ocular hypertension. Acta Ophthalmol 1981;59:627-41.

27 Jonas JB, Nguyen NX, Naumann GOH. The retinal nerve fiber layer in normal eyes. Ophthalmology 1989;96:627-32.

28 Quigley HA, Addicks EM. Regional differences in the structure of the lamina cribrosa and their relation to glaucomatous optic nerve damage. Arch Ophthalmol 1981;99:13743.

29 Jonas JB, Dichtl A. Evaluation of the retinal nerve fiber layer. Surv Ophthalmol 1996;40:369-78.

30 Quigley HA, Addicks EM. Quantitative studies of retinal nerve fiber layer defects. Arch Ophthalmol 1982;100:80714.

31 Radius RL, Anderson DR The histology of the retinal nerve fiber bundles and bundle defects. Arch Ophthalmol 1979;97:948-50.

32 Dichtl A, Jonas JB, Naumann GOH. Retinal nerve fiber layer thickness in human eyes. Graefes Arch Clin Exp Ophthalmol 1999;237:474-9. 